\title{
Diabetes medications with cardiovascular protection in the wake of EXSCEL: is there a class effect for long-acting GLP-1 receptor agonists?
}

\author{
ROBERT EJ RYDER, ${ }^{1}$ RALPH A DEFRONZO²
}

In previous editorials ${ }^{1-3}$ we proposed that metformin, pioglitazone, sodium glucose transporter 2 (SGLT2) inhibitors (in particular empagliflozin and canagliflozin) and liraglutide in combination could complement each other to prevent cardiovascular events and save lives in patients with type 2 diabetes at high cardiovascular risk. In published trials with glucagon-like peptide-1 (GLP-1) receptor agonists, cardiovascular benefit has been shown for the long-acting agents liraglutide (LEADER ${ }^{4}$ ) and semaglutide (SUSTAIN 65), although not for the short-acting agent lixisenatide (ELIXA6). In the light of this there has been particular interest in the result of the EXenatide Study of Cardiovascular Event Lowering (EXSCEL) with once-weekly exenatide (exenatide QW), as this could potentially give insight into whether there was a class effect with regard to long-acting GLP-1 receptor agonists. Thus, for many healthcare professionals who care for patients with diabetes, there was some disappointment when, on 23 May 2017, AstraZeneca issued a press release announcing that, whilst EXSCEL had met its primary safety objective, "the efficacy objective of superior reduction in Major Adverse Cardiovascular Events (MACE; a composite end point of cardiovascular death, non-fatal myocardial infarction and nonfatal stroke) did not reach statistical significance". ${ }^{7}$ Nevertheless, the full results were awaited with interest.

On 14 September 2017, during the European Association for the Study of Diabetes congress in Lisbon, Portugal, the results of EXSCEL were presented ${ }^{8}$ and published simultaneously in the New England Journal of Medicine. ${ }^{9}$ The atmosphere in the auditorium was noticeably different from that of other similar presentations of cardiovascular outcome studies: EMPA-REG, ${ }^{1}$ LEADER, ${ }^{2}$ SUSTAIN $6{ }^{10}$ and, most recently, CANVAS. ${ }^{3}$ This was because there were no slides showing significantly reduced cardiovascular death or significantly reduced 3-point MACE (cardiovascular death, non-

City Hospital, Birmingham, UK

University of Texas Health Science Center, San Antonio, Texas, USA

Address for correspondence: Dr Bob Ryder

Diabetes and Endocrine Unit, City Hospital, Dudley Road, Birmingham B18 7QH, UK

E-mail: bob.ryder@nhs.net

Br J Diabetes 2017; 17:131-133

https://doi.org/10.15277/bjd.2017.147 fatal myocardial infarction and non-fatal stroke) to be greeted with enthusiastic applause by a large enthralled audience. The study was large (14,752 patients) with high cardiovascular risk and yet the primary composite end point, 3-point MACE, the same as that in LEADER and SUSTAIN 6, did not achieve statistical significance (hazard ratio (HR) 0.91; 95\% confidence interval (Cl) 0.83 to 1, $\mathrm{p}=0.06) \cdot 8,9$

Another cardiovascular outcome study with a long-acting exenatide-based medication was FREEDOM-CVO with ITCA 650. ${ }^{11}$ At first sight the top-line results seem similar to those of EXSCEL in apparently showing non-inferiority but not superiority. ${ }^{11}$ However, FREEDOM-CVO recruited only about 4000 patients solely for the purpose of fulfilling the pre-approval cardiovascular safety outcome required by the US Food and Drug Administration (FDA). A separate trial dedicated to demonstrating possible cardiovascular benefit for ITCA 650 is under consideration. ${ }^{11}$ Nevertheless, it is noteworthy that the amino acid structure differs between exendin-4 and native GLP-1. ${ }^{8}$ Could this be a factor in the fact that it was exendin-based GLP-1 receptor agonists used in the trials with apparently negative outcomes (ELIXA, EXSCEL, FREEDOM-CVO) while the trials based on GLP-1-based GLP-1 receptor agonists (LEADER, SUSTAIN 6) were positive? Professor Francesco Giorgino, in his commentary on EXSCEL, ${ }^{8}$ acknowledged the amino acid difference and some evidence to suggest that there is potential for differences in the signalling of the different GLP-1 receptor agonists. However, he then pointed to multiple studies in humans examining endpoints relevant to cardiovascular protection that have demonstrated that liraglutide and exenatide have similar properties in this regard. ${ }^{8}$

A more detailed look at the results of EXSCEL suggest that there is, after all, a class effect for long-acting GLP-1 receptor agonists. Of note, the $p$ value of 0.06 closely approximates statistical significance and the hazard ratio of 0.91 is close to that of LEADER (0.87).

Comparison of EXSCEL and LEADER, the two largest cardiovascular studies involving GLP-1 receptor agonists, demonstrates significant differences between them that could explain why one (LEADER) achieved statistical significance while the other (EXSCEL) fell slightly short of its goal. The LEADER study population was at higher cardiovascular risk than the EXSCEL population. ${ }^{8}$ Both studies included a primary prevention cohort as well as a secondary prevention cohort, the latter being at higher cardiovascular risk, already having experienced an event. In LEADER only $19 \%$ of participants 
Figure 1. Primary endpoint and its individual components and all-cause mortality in LEADER and EXSCEL. Number needed to treat to save one life was 98 for 3 years in LEADER and 106 for 3 years in EXSCEL

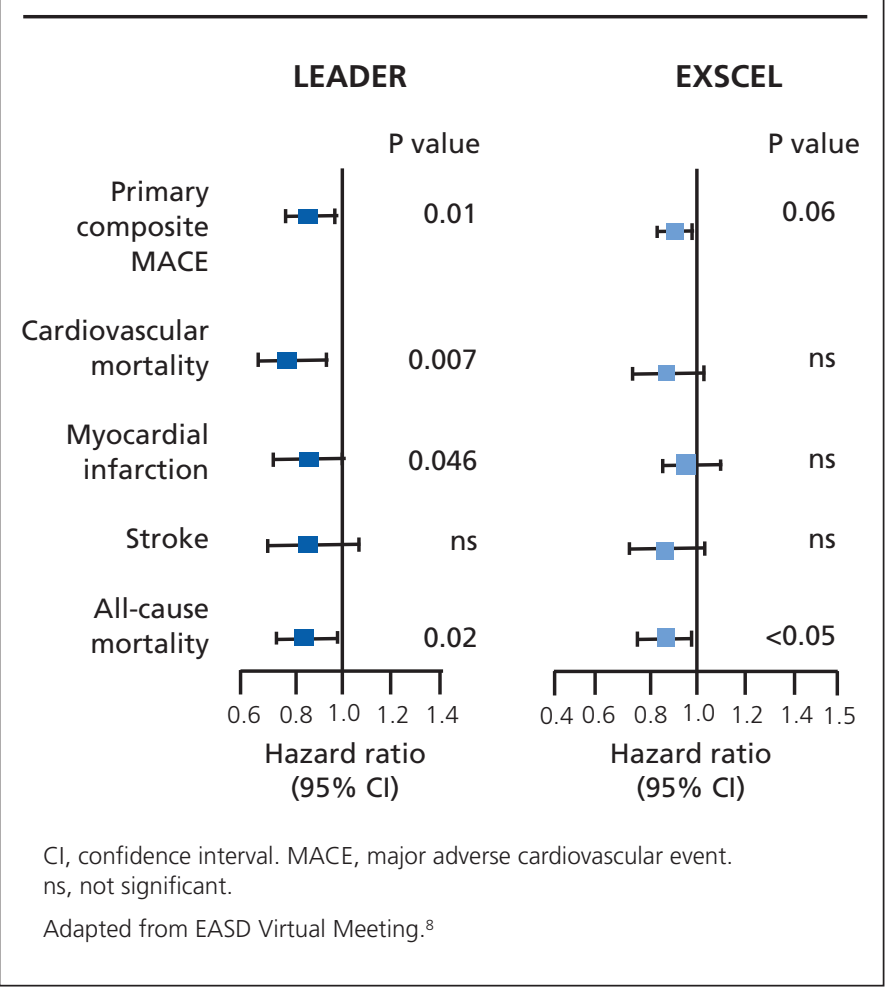

were primary prevention, whereas in EXSCEL $27 \%$ had yet to have a cardiovascular event. ${ }^{8}$ The LEADER patients had poorer glycaemic control: mean baseline $\mathrm{HbA}_{1 \mathrm{c}}$ in LEADER was $8.7 \%$ whereas in EXSCEL it was $8.1 \% .^{8}$ The median study drug exposure in LEADER was 3.5 years whereas in EXSCEL it was only 2.4 years. ${ }^{8}$ Perhaps, most importantly, in EXSCEL there was premature discontinuation of the study drug in $43 \%$ of the patients. ${ }^{8}$ Possible factors contributing to this high discontinuation rate were concerns about the safety of GLP-1 receptor agonists, in particular with regard to pancreatitis and pancreatic cancer, which received wide media attention whilst EXSCEL was in progress. ${ }^{12}$ Another possible factor contributing to the high discontinuation rate may have been the fact that the early device used for administering exenatide QW was not particularly easy to use. ${ }^{8}$ Further, the use of all classes of antiglycaemic medications, including those known to improve cardiovascular outcomes (metformin, pioglitazone, SGLT2 inhibitors and GLP-1 receptor agonists) was higher in the placebo $\mathrm{arm}^{8}$ and this may have improved cardiovascular outcomes in the placebo arm. ${ }^{1-3}$

In Figure 1, the major LEADER and EXSCEL outcomes are compared side by side. The similarity in the pattern of the point estimates is noteworthy. This raises the possibility - indeed likelihood - that the two agents have similar effects, but in EXSCEL they did not achieve the statistical significance achieved in LEADER because of the differences in the studes that we have pointed out. After drawing our attention to these differences, Professor Rury Holman in his overview 'EXSCEL in perspective' stated: "We cannot exclude

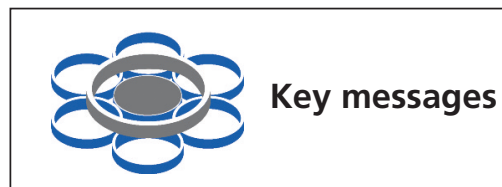

- In the EXSCEL study, exenatide QW showed a reduction in 3-point MACE (cardiovascular death, non-fatal myocardial infarction and non-fatal stroke) compared with placebo, but this did not achieve statistical significance.

- A comparison between the LEADER (liraglutide) and EXSCEL (exenative QW) cardiovascular outcome studies raises the possibility that the difference in outcomes with regard to statistical significance might be related to the fact that the LEADER patients were at higher cardiovascular risk and had longer exposure to study medication than the EXSCEL patients, due to a high discontinuation rate in EXSCEL.

- Comparing the point estimates from the two studies side by side suggests that the results are more similar than dissimilar, and are in keeping with a class effect for long-acting GLP-1 receptor agonists, which might have been more robustly confirmed if the patients in EXSCEL had higher cardiovascular risk and greater exposure to study medication.

- Nevertheless, when choosing a long-acting GLP-1 receptor agonist for high cardiovascular risk patients, it would be prudent to choose the definitely proven medication (liraglutide) above the unproven one (exenatide QW).

that there are differences at the molecular level for these agents, but we believe that the data we have seen with the length of follow-up and these differences suggest the class as a whole are consistent in their effects on cardiovascular outcomes". 8

In keeping with the suggestion that a contributing factor to the non-significant primary outcome of the EXSCEL trial was related to the study population not being at high enough cardiovascular risk was the finding that the 3-point MACE endpoint was significantly reduced in those aged over 65 years (HR 0.80; 95\% Cl 0.71 to $0.91, p=0.005)$, which is of interest bearing in mind that older people are likely to be at higher risk. ${ }^{8}$

In conclusion, taking into consideration the positive outcomes of LEADER ${ }^{2,10}$ and SUSTAIN 65,10 with regard to cardiovascular benefit from long-acting GLP-1 receptor agonist therapy in patients at high risk of cardiovascular disease, the results from EXSCEL, in particular when the point estimates for LEADER and EXSCEL are compared side by side (Figure 1), are compatible with a class effect for long-acting GLP-1 receptor agonists. The non-significant results in EXSCEL could be related to differences between the studies, in particular the LEADER patients starting at higher cardiovascular risk and exposure to the study drug in EXSCEL being only two-thirds of that in LEADER. ${ }^{8}$ If there is indeed such a class effect of cardiovascular benefit for long-acting GLP-1 receptor agonists, we would 
expect positive outcomes from sufficiently large, appropriately designed, cardiovascular outcome studies with both exendin-4 based GLP-1 receptor agonists (such as should be conducted with ITCA 65011) and that which is being conducted with another GLP1-based GLP-1 receptor agonist, dulaglutide (the REWIND study'3). In the meantime, for clinicians, when it comes to the choice of available agent from the long-acting GLP-1 class to treat the patient at high cardiovascular risk, the agent with proven cardiovascular benefit (liraglutide) surely has to be chosen above the one that is unproven (exenatide QW). In view of this, it is timely that the European Medicines Agency has recently changed its summary of product characteristics with regard to liraglutide to incorporate a description of the LEADER results and to acknowledge to the public that liraglutide has been "shown to be effective at reducing adverse cardiovascular (heart and blood vessels) effects". ${ }^{14}$

Conflict of interest REJR: speaker fees and/or consultancy fees and/or educational sponsorships from AstraZeneca, BioQuest, GI Dynamics, Janssen, Novo Nordisk, Sanofi-Aventis and Takeda. RAD: Advisory board: AstraZeneca, Novo Nordisk, Janssen, Lexicon, Boehringer-Ingelheim; Research support: Bristol-Myers Squibb, Boehringer-Ingelheim, Takeda, AstraZeneca; Speaker's bureau: Novo Nordisk, AstraZeneca.

\section{References}

1. Ryder REJ, DeFronzo RA. Diabetes medications with cardiovascular protection in the wake of EMPA-REG OUTCOMETM: the optimal combination may be metformin, pioglitazone and empagliflozin. $\mathrm{Br} J$ Diabetes Vasc Dis 2015;15:151-4. http://dx.doi.org/10.15277/bjdvd.2015.045

2. Ryder REJ, DeFronzo RA. Diabetes medications with cardiovascular protection - what now after LEADER ${ }^{\circledR}$ ? Could metformin, pioglitazone, empagliflozin and liraglutide complement each other to save lives? $\mathrm{Br} J$ Diabetes 2016;16:103-06. http://dx.doi.org/10.15277/bjd.2016.096

3. Ryder REJ, DeFronzo RA. What now on the CANVAS of diabetes medications with cardiovascular protection? Could metformin, pioglitazone, SGLT2 inhibitors and liraglutide complement each other to save lives? $\mathrm{Br}$ J Diabetes 2017;17:89-92. http://dx.doi.org/10.15277/bjd.2017.036

4. Marso SP, Daniels GH, Brown-Frandsen K, et al, LEADER Steering Committee; LEADER Trial Investigators. Liraglutide and cardiovascular outcomes in type 2 diabetes. N Engl J Med 2016;375:311-22. http://dx.doi.org/10.1056/NEJMoa1603827

5. Marso SP, Bain SC, Consoli A, et al, SUSTAIN-6 Investigators. Semaglutide and cardiovascular outcomes in patients with type 2 diabetes. $N$ Engl $J$ Med 2016;375:1834-44. http://dx.doi.org/10.1056/NEJMc1615712

6. Pfeffer, MA, Claggett, B, Diaz, R, et al. Lixisenatide in patients with type 2 diabetes and acute coronary syndrome. N Engl J Med 2015;373:224757. http://dx.doi.org/10.1056/NEJMoa1509225

7. AstraZeneca. Bydureon EXSCEL trial meets primary safety objective in type-2 diabetes patients at wide range of cardiovascular risk. Press release 23 May 2017. https://www.astrazeneca.com/media-centre/pressreleases/2017/bydureon-exscel-trial-meets-primary-safety-objective-intype-2-diabetes-patients-at-wide-range-of-cardiovascular-risk23052017.html (accessed 12 October 2017).

8. EASD Virtual Meeting. Webcast: EXenatide Study of Cardiovascular Event Lowering (EXSCEL): primary results. https://www.easd.org/virtualmeeting/home.html\#! contentsessions/2628 (accessed 1 November 2017).

9. Holman RR, Bethel MA, Mentz RJ, et al, EXSCEL Study Group. Effects of once-weekly exenatide on cardiovascular outcomes in type 2 diabetes. N Engl J Med 2017;377:1228-39. http://dx.doi.org/10.1056/NEJMoa1612917

10. Abdul-Ghani M, DeFronzo RA, Del Prato S, Chilton R, Singh R, Ryder REJ. Cardiovascular disease and type 2 diabetes: has the dawn of a new era arrived? Diabetes Care 2017;40:813-20. https://doi.org/10.2337/dc16-2736

11. Intarcia. Intarcia announces successful cardiovascular safety results in phase 3 FREEDOM-CVO trial for ITCA 650, an investigational therapy for type 2 diabetes. Press release 6 May 2016. https://www.intarcia.com/ media/press-releases/2016-may-6-cardiovascular-safety.html (accessed 1 November 2017).

12. Ryder REJ. The potential risks of pancreatitis and pancreatic cancer with GLP-1-based therapies are far outweighed by the proven and potential (cardiovascular) benefits. Diabet Med 2013;30:1148-55. https://doi.org/10.1111/dme.12301

13. Gerstein HC, Colhoun HM, Dagenais GR, et al. Design and baseline characteristics of participants in the Researching cardiovascular Events with a Weekly INcretin in Diabetes (REWIND) trial on the cardiovascular effects of dulaglutide. Diabetes Obes Metab 1 June 2017. doi:10.1111/dom.13028. [Epub ahead of print]

14. European Medicines Agency. Victoza (liraglutide). Summary of product characteristics. Update 14/09/2017. http://www.ema.europa.eu/docs/ en_GB/document_library/EPAR_-_Product_Information/human/ 001026/WC500050017.pdf (accessed 12 October 2017). 